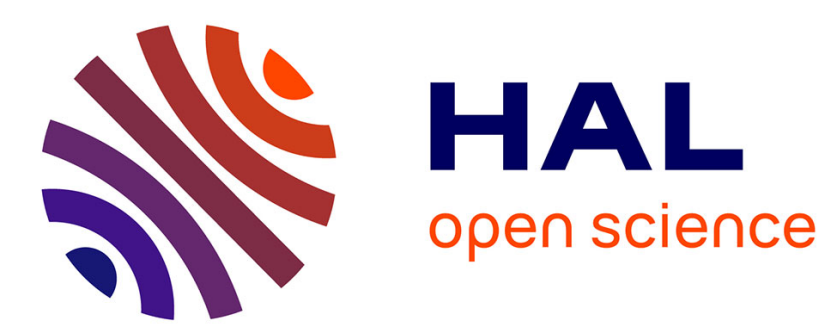

\title{
Exact properties of spin glasses. - I. 2D supersymmetry and Nishimori's result
}

A. Georges, D. Hansel, P. Le Doussal

\section{To cite this version:}

A. Georges, D. Hansel, P. Le Doussal. Exact properties of spin glasses. - I. 2D supersymmetry and Nishimori's result. Journal de Physique, 1985, 46 (8), pp.1309-1317. 10.1051/jphys:019850046080130900 . jpa-00210074

\section{HAL Id: jpa-00210074 https://hal.science/jpa-00210074}

Submitted on 1 Jan 1985

HAL is a multi-disciplinary open access archive for the deposit and dissemination of scientific research documents, whether they are published or not. The documents may come from teaching and research institutions in France or abroad, or from public or private research centers.
L'archive ouverte pluridisciplinaire HAL, est destinée au dépôt et à la diffusion de documents scientifiques de niveau recherche, publiés ou non, émanant des établissements d'enseignement et de recherche français ou étrangers, des laboratoires publics ou privés. 
Classification

Physics Abstracts

$75.40-05.50$

\title{
Exact properties of spin glasses. I. 2D supersymmetry and Nishimori's result
}

\author{
A. Georges $\left({ }^{+}\right)$, D. Hansel $\left(^{++}\right)$and P. Le Doussal $\left(^{+}\right)$ \\ $\left({ }^{+}\right)$Laboratoire de Physique Théorique de l'Ecole Normale Supérieure $\left({ }^{*}\right), 24$, rue Lhomond, 75231 Paris Cedex 05, \\ France \\ $\left(^{++}\right)$Centre de Physique Théorique de l'Ecole Polytechnique $\left(^{* *}\right)$, route de Saclay, 91128 Palaiseau Cedex, \\ France
}

(Reçu le 19 mars 1985, accepté le 19 avril 1985)

\begin{abstract}
Résumé. - Nous proposons une théorie effective de fermions et bosons en interaction afin d'exprimer l'énergie interne du verre de spin d'Ising bidimensionnel. Nous montrons qu'un résultat exact obtenu par Nishimori s'interprète, dans cette formulation, comme une réduction dimensionnelle due à l'apparition d'une supersymétrie. Ceci conduit à une meilleure compréhension de ce résultat exact pour un verre de spin d'Ising général.
\end{abstract}

\begin{abstract}
We introduce an effective theory of interacting fermions and bosons in order to express the quenched internal energy of the 2D Ising spin glass. We show that an exact result derived by Nishimori appears, in this formulation, as a dimensional reduction due to the apparition of a supersymmetry. For a general Ising spin glass, this suggests new insights into the physical meaning of this exact result.
\end{abstract}

\section{Introduction.}

The physics of spin glasses has received considerable interest since the pioneering paper by Edwards and Anderson [1]. As in all disordered systems, the main theoretical difficulty is the evaluation of quenched averages. In order to tackle this problem, one generally makes use of the replica trick, introduced by Edwards and Anderson [1]. Moreover, in other disordered problems, such as localization theory, other methods based on some fermion-boson symmetry [2-4] have been introduced.

In this paper, we propose such a method to study the Ising spin glass in two dimensions with nearest neighbour interactions. For the corresponding pure model, it is well known that free fermion formulations of the partition function are available. This allows us to express the quenched internal energy of the model as a path integral over bosonic and fermionic fields.

$\left(^{*}\right)$ Laboratoire Propre du Centre National de la Recherche Scientifique associé à l'Ecole Normale Supérieure et à l'Université de Paris-Sud.

$\left({ }^{* *}\right)$ Groupe de Recherche du Centre National de la Recherche Scientifique no 48.
Apart from possibly providing a new technique to study the 2D Ising spin glass, this method proves to be useful in the understanding of an exact result derived by Nishimori $[5,6]$ for a general Ising spin glass. We now briefly summarize this result. Defining $P\left(J_{i j}\right)$ as the probability distribution over the bonds, the relation :

$$
\frac{P\left(-J_{i j}\right)}{P\left(J_{i j}\right)}=\mathrm{e}^{-2 \beta J_{i j}}
$$

defines a subspace of the phase diagram. Nishimori showed that, on this subspace, the quenched internal energy of the model simplifies drastically to become :

$$
U=-N_{\mathrm{B}} \int J P(J) \mathrm{d} J=-N_{\mathrm{B}}\langle J\rangle
$$

( $N_{\mathrm{B}}$ is the number of bonds.)

In this paper, we show that this remarkable result can be understood as a dimensional reduction due to a restoration of the fermion-boson symmetry (supersymmetry) in our expression of the quenched internal energy. This leads us to introduce a function which controls this restoration and can be calculated exactly on Nishimori's subspace. This function has a simple 
physical interpretation, even in higher dimensions where no fermion-boson formulation is available. This provides new physical insights into Nishimori's result.

This paper is organized as follows. In section 2, we derive the fermion-boson formulation for $U$. In section 3 , we show how supersymmetry is restored on Nishimori's subspace. Finally, in section 4, we discuss the physical interpretation of Nishimori's result for a general Ising spin glass.

In a companion paper, we will generalize Nishimori's result to any gauge invariant spin glass. We will also obtain information on phase diagrams by comparing these exact results with existing numerical and theoretical works.

\section{Fermion-boson expression of the quenched energy.}

In this section, we propose a method avoiding the replica trick to perform the quenched average in the expression of the internal energy :

$$
U=-\left\langle\frac{1}{Z} \frac{\partial Z}{\partial \beta}\right\rangle .
$$

$Z$ is the partition function of the model, and the bracket denotes the average over the random bonds. When one takes this average, the difficulty comes from the factor $1 / Z$ in (2.1). We notice that when $Z$ can be expressed as a Gaussian integral over fermionic variables, $1 / Z$ is the corresponding Gaussian integral over bosonic variables. This eliminates the denominator, and the quenched average can now be easily performed. We thus obtain an interacting theory of bosons and fermions.

We first illustrate this general idea by the very simple example of the one-dimensional Ising spin glass with nearest neighbour interactions. We then turn to the more involved case of dimension two.

2.1 The ONE-DIMENSIONAL CASE. - We consider a chain of $N$ Ising spins $S_{i}$, with cyclic boundary conditions $S_{N+1}=S_{1}$. The Hamiltonian of the model is :

$$
\mathscr{H}=-\sum_{i=1}^{N} J_{i, i+1} S_{i} S_{i+1}
$$

and the partition function, for a given configuration of bonds, reads :

$$
Z=Z_{\mathrm{R}}\left(1+\prod_{k=1}^{N} \tanh \beta J_{k, k+1}\right)
$$

where :

$$
Z_{\mathrm{R}}=2^{N} \prod_{i=1}^{N} \cosh \beta J_{i, i+1}
$$

(In the following, we set $t_{i}=\tanh \beta J_{i, i+1}$.) In the thermodynamic limit the contribution of the $t_{k}$ 's vanishes, which is a special feature of dimension 1 . However, it will be of importance in section 3 to consider a formulation also valid for finite size systems.
When $N$ is odd, (2.3) is a determinant. Hence, introducing two Grassmann variables $x_{i}, x_{i}^{+}$at each site, it can be written as :

$$
Z=Z_{\mathbf{R}} \int D F \exp S_{\mathrm{F}}
$$

with :

$$
S_{\mathrm{F}}=-\sum_{i=1}^{N}\left(t_{i} x_{i}^{+} x_{i+1}+x_{i}^{+} x_{i}\right)
$$

and where $D F$ denotes $\prod_{i=1}^{N} \mathrm{~d} x_{i} \mathrm{~d} x_{i}^{+}$.

In the same way, one can write $Z^{-1}$ as an integral over bosonic site variables $\chi_{i}, \chi_{i}^{*}$ :

$$
\begin{gathered}
Z^{-1}=Z_{\mathrm{R}}^{-1} \int D B \exp S_{\mathrm{B}} \\
S_{\mathrm{B}}=-\sum_{i=1}^{N}\left(t_{i} \chi_{i}^{*} \chi_{i+1}+\chi_{i}^{*} \chi_{i}\right) .
\end{gathered}
$$

We are now ready to write the quenched internal energy of the corresponding random bond model in the way explained above. Denoting by $\mathscr{D} P=\prod_{i=1}^{N}$ $P\left(J_{i, i+1}\right) \mathrm{d} J_{i, i+1}$ the probability measure over the bonds, we obtain :

$$
U=-N\langle J \tanh \beta J\rangle+U_{\mathrm{s}}
$$

where

$$
U_{\mathrm{S}}=N \int D F D B \int \mathcal{D P} J_{1,2}\left(1-t_{1}^{2}\right) x_{1}^{+} x_{2} \mathrm{e}^{S_{\mathrm{F}}+S_{\mathrm{B}}}
$$

We have used translational invariance after averaging. This expression does not involve any denominator : the integral over $D P$ splits into a product of single integrals, and the average can be performed, at least formally. Defining the effective action $S_{\text {eff }}$ as :

$$
\mathrm{e}^{S_{\text {eff }}(\mathbf{F}, \mathbf{B})}=\int \mathcal{D P J}_{1,2}\left(1-t_{1}^{2}\right) \mathrm{e}^{S_{\mathbf{F}}+S_{\mathbf{B}}}
$$

we obtain :

$$
U_{\mathrm{S}}=N \int D F D B x_{1}^{+} x_{2} \mathrm{e}^{S_{\text {erf }}(\mathbf{F}, \mathbf{B})}
$$

This expresses $U_{\mathrm{s}}$ as the mean value of the operator $x_{1}^{+} x_{2}$ in the theory of interacting fermions and bosons defined by $S_{\text {eff }}$. This action is clearly invariant under the exchange of fermionic and bosonic variables, that is under the following "supersymmetry » transformation :

$$
\begin{array}{ll}
\delta x_{i}=\varepsilon \chi_{i} & \delta \chi_{i}=-\varepsilon^{+} x_{i} \\
\delta x_{i}^{+}=\varepsilon^{+} \chi_{i}^{*} & \delta \chi_{i}^{*}=-x_{i}^{+} \varepsilon
\end{array}
$$

where $\varepsilon, \varepsilon^{+}$are Grassmannian parameters. Let's 
emphasize that the operator $x_{1}^{+} x_{2}$ is obviously not invariant under this symmetry : this will be of importance later.

We now turn to the case of dimension two.

2. 2 THE TWO-DIMENSIONAL CASE. - We consider here the Ising model with nearest neighbour interactions on the $2 \mathrm{D}$ Ising square lattice :

$$
\mathcal{H}=-\sum_{k=1}^{N} \sum_{\mathbf{u}=1,2} J_{k, k+\mathrm{u}} S_{k} S_{k+\mathbf{u}}
$$

where $\mathbf{u}=1,2$ are the two lattice vectors. The first step to be taken is to write the partition function for a given configuration of bonds as an integral over fermionic variables. Several formulations are available which all use the fact that $Z$ is a Pfaffian (the square-root of the determinant of an antisymmetric matrix). This does not allow us to write $Z^{-1}$ as the corresponding Gaussian integral over complex bosonic variables, as this trick only applies to a determinant. (The use of real variables does not help, as it applies to symmetric matrices only.) This difficulty can be overcome by using $Z^{2}$, which is a determinant, instead of $Z$, and by noting that :

$$
U=-\frac{1}{2}\left\langle\frac{1}{Z^{2}} \frac{\partial}{\partial \beta}\left(Z^{2}\right)\right\rangle .
$$

This amounts to a doubling of the number of fermions at each site.

To be specific, we choose to use four independent fermions at each site (which become eight after doubling). All the relevant expressions and transformations are given in Appendix A. Let us only summarize the form we obtain for $Z^{2}$ :

$$
Z^{2}=Z_{\mathrm{R}}^{2} \int D F \mathrm{e}^{S_{F}}
$$

where $D F$ denotes the complete Grassmann lattice measure.

As in dimension one, $Z_{\mathbf{R}}$ is the part of the partition function given by :

$$
Z_{\mathbf{R}}=2^{N} \prod_{k, \mathbf{u}} \cosh \beta J_{k, k+\mathbf{u}}
$$

which only leads to regular contributions in the thermodynamic limit.

The fermionic action in (2.16) can be written :

$$
S_{\mathrm{F}}=\sum_{k=1}^{N}\left(t_{k, k+1} K_{k, k+1}^{\mathrm{F}}+t_{k, k+2} K_{k, k+2}^{\mathrm{F}}+V_{k}^{\mathrm{F}}\right)
$$

In this formula, the only dependence on the random bonds lies in $t_{k, k+u}=\tanh \beta J_{k, k+u} . K^{\mathrm{F}}$ and $V^{\mathrm{F}}$ are quadratic operators in the fermion fields : $K_{k, k+a}$ couples two nearest neighbour sites and is thus a kinetic operator, while $V_{k}$ is a local potential.
Using (2.15), we obtain a similar expression to the one we obtain in dimension one for the quenched internal energy :

$$
U=-N_{\mathbf{B}}\langle J \tanh \beta J\rangle+U_{\mathrm{S}}
$$

where $N_{\mathrm{B}}$ is the number of bonds, and :

$$
\begin{array}{r}
U_{\mathrm{S}}=\frac{N}{2} \int D F D B \int \mathcal{D} P \sum_{\mathbf{u}} J_{0,0+\mathbf{u}}\left(1-t_{0,0+\mathbf{u}}^{2}\right) \times \\
\times K_{0,0+\mathbf{u}}^{\mathrm{F}} \mathrm{e}^{S_{\mathbf{F}}+S_{\mathrm{B}}} .
\end{array}
$$

We have assumed appropriate boundary conditions, in such a way that, after averaging, all sites are equivalent to a given one, denoted by 0 .

As in dimension one, $U_{\mathrm{S}}$ is expressed as the mean value of a non supersymmetric operator in a supersymmetric effective theory of interacting bosons and fermions. Though it is not our purpose in this paper, it would be extremely interesting to obtain physical information on the 2D spin glass by using this effective theory.

In particular, one can hope to confirm the results of Vik. S. Dotsenko and V. I. Dotsenko [7] concerning the critical properties of the paramagnetic/ferromagnetic transition. This could perhaps be achieved by a renormalization group study of the fermion-boson field theory obtained from our effective theory by taking the continuous limit.

Another interesting point to be studied is the behaviour of the ground state of this effective theory under supersymmetry.

Let us close this section by a remark concerning the use of such " supersymmetric " methods in disordered problems. The term "supersymmetry » in fact refers to two very different classes of methods.

The first one, based on the trick used in this paper, leads to an elementary form of supersymmetry which amounts simply to the exchange of commuting and anticommuting variables (see (2.13)). In particular, these variables have the same kinetic terms (same " spin ") : this does not correspond to what is called " supersymmetry » in particle physics. This first kind of methods was introduced by MacKane [2] and Parisi and Sourlas [3] and is used in the polymer problem and in localization theory [4].

Another class of methods, which are true supersymmetries in the sense of particle physics, was introduced by Parisi and Sourlas for the problems of random magnetic fields [8] and stochastic equations [9].

\section{Nishimori's line, supersymmetry and dimensional reduction.}

In this section we show that Nishimori's exact result stated in the introduction has a simple interpretation in the supersymmetric formulation of section 2 .

3.1 RESTORING SUPERSYMMETRY. - As in formulae (2.9) and (2.19), let us split the quenched internal 
energy into a regular contribution and a non trivial one (potentially singular in the thermodynamic limit) :

$$
U=-N_{\mathrm{B}}\langle J \tanh \beta J\rangle+U_{\mathrm{S}} .
$$

We notice that on Nishimori's line defined by :

$$
\frac{P(-J)}{P(J)}=\mathrm{e}^{-2 \beta J}
$$

two remarkable simplifications occur :

i) $U_{\mathrm{S}}$ vanishes ;

ii) $-N_{\mathrm{B}}\langle J \tanh \beta J\rangle=-N_{\mathrm{B}}\langle J\rangle$.

We will show that the simplification i) can be understood as the restoration of supersymmetry of the operator associated with $U_{\mathrm{S}}$ in the path integral formulation (2.10) and (2.20).

Let us begin with the expression (2.10) of $U_{\mathrm{S}}$ in one dimension. Using the identity valid for quadratic actions :

$$
\int D F D B \mathrm{e}^{S_{F}+S_{B}}=1
$$

We can put (2.10) under the equivalent form :

$$
\begin{aligned}
U_{\mathrm{S}}=\frac{N}{2} \int D F D B \int & \operatorname{DPJ}_{12}\left(1-t_{1}^{2}\right) \times \\
& \times\left(x_{1}^{+} x_{2}-\chi_{1}^{*} \chi_{2}\right) \mathrm{e}^{S_{\mathrm{F}}+S_{\mathrm{B}}} .
\end{aligned}
$$

When integrating over $\mathfrak{D P}$, we make the change $J_{12} \rightarrow-J_{12}$ in the term involving $\chi_{1}^{*} \chi_{2}$. This leads to :

$$
\begin{aligned}
& U_{\mathrm{S}}=\frac{N}{2} \int D F D B \int \mathcal{D P J} J_{12}\left(1-t_{1}^{2}\right) \times \\
& \times\left(x_{1}^{+} x_{2}+\rho_{N}\left(J_{12}\right) \frac{P\left(-J_{12}\right)}{P\left(J_{12}\right)} \chi_{1}^{*} \chi_{2}\right) \mathrm{e}^{S_{F}+S_{B}}
\end{aligned}
$$

where we have defined the function $\rho_{N}\left(J_{12}\right)$ as the ratio of two quantities averaged over all bonds except one which is frozen to $\pm J_{12}$ (such an average is denoted by \langle\rangle$\left.^{*}\right)$ :

$\rho_{N}\left(J_{12}\right)=\frac{\left\langle\int D F D B \chi_{1}^{*} \chi_{2} \mathrm{e}^{S_{\mathrm{F}}\left(-t_{1}\right)+S_{\mathrm{B}}\left(-t_{1}\right)}\right\rangle^{*}}{\left\langle\int D F D B \chi_{1}^{*} \chi_{2} \mathrm{e}^{S_{\mathrm{F}}\left(t_{1}\right)+S_{\mathrm{B}}\left(t_{1}\right)}\right\rangle^{*}}$.

We thus see that as soon as the following condition is met :

$$
\rho_{N}(J) \frac{P(-J)}{P(J)}=1
$$

the integral (3.5) is automatically zero (by taking the derivative of (3.3) relatively to $\beta$ ).

We will show in subsection 3.2 that this is actually what happens on Nishimori's line. This restores the supersymmetry of the operator associated with $U_{\mathrm{S}}$ in the effective theory. Using the expression (2.20) of $U_{\mathrm{S}}$, all these manipulations can be readily generalized to dimension 2 . The supersymmetry of the operator is restored and $U_{S}$ vanishes when condition (3.7) is met; $\rho_{N}(J)$ is defined here by :

$=\frac{\left\langle\int D F D B K_{0,0+1}^{\mathrm{B}} \mathrm{e}^{\rho_{\mathrm{F}}\left(-t_{0,0+1}\right)+S_{\mathrm{B}}\left(-t_{0,0+1}\right)}\right\rangle^{*}}{\left\langle\int D F D B K_{0,0+1}^{\mathrm{B}} \mathrm{e}^{\left.S_{\mathrm{F}\left(t_{0}, 0+1\right.}\right)+S_{\mathrm{B}}\left(t_{0,0+1}\right)}\right\rangle^{*}}$

each direction and each site of the lattice being equivalent.

Apart from illustrating how Nishimori's simplification works in a supersymmetric formulation, these results will be shown in section 4 to have a simple physical interpretation. This interpretation is based on a generalization of the function $\rho_{N}(J)$ to higher dimensional Ising models (where no supersymmetric formulation is available).

3.2 Simplification of $\rho_{N}(J)$ ON Nishimori's Line. The function $\rho_{N}$ depends on the probability distribution $P$, on the size $N$ of the system and has a priori the full complexity of the original problem. Nevertheless, on Nishimori's line defined by (3.2), it simplifies drastically to become :

$$
\rho_{N}(J)=\mathrm{e}^{2 \beta J}
$$

in such a way that condition (3.7) be satisfied on the line.

Let us first prove this statement in dimension 1, where explicit expressions are available and where the mechanism of this simplification appears clearly. Indeed the Gaussian integral in (3.6) can be performed to obtain :

$$
\rho_{N}(J)=\frac{\int \mathscr{D} P^{*} \frac{t_{2} \ldots t_{N}}{1-t_{1} \ldots t_{N}}}{\int \mathfrak{D P} P^{*} \frac{t_{2} \ldots t_{N}}{1+t_{1} \ldots t_{N}}}
$$

with $\mathfrak{D} P^{*}=\prod_{i=2}^{N} P\left(J_{i, i+1}\right) \mathrm{d} J_{i, i+1}$.

Expanding the ratios and taking the average leads us to :

$\rho_{N}\left(J_{1}\right)=$
$=\frac{\sum_{p=0}^{\infty} t_{1}^{2 p}\left\langle t^{2 p+1}\right\rangle^{N-1}+\sum_{p=0}^{\infty} t_{1}^{2 p+1}\left\langle t^{2 p+2}\right\rangle^{N-1}}{\sum_{p=0}^{\infty} t_{1}^{2 p}\left\langle t^{2 p+1}\right\rangle^{N-1}-\sum_{p=0}^{\infty} t_{1}^{2 p+1}\left\langle t^{2 p+2}\right\rangle^{N-1}}$.

We notice that on Nishimori's line one has :

$$
\left\langle t^{2 p+1}\right\rangle=\left\langle t^{2 p+2}\right\rangle \text {. }
$$


Hence, a surprising factorization occurs :

$$
\rho_{N}\left(J_{1}\right)=\frac{1+t_{1}}{1-t_{1}}
$$

which is nothing but (3.9).

In two dimensions, no explicit expression such as (3.11) can be given. Nevertheless, in Appendix B we give a general proof of (3.9) based on gauge invariance. This proof is given there using an equivalent formulation of $\rho_{N}(J)$ in terms of the original Ising spin variables, which allows us to generalize $\rho_{N}(J)$ to higher dimensions, as shown in section 4.

We thus see that, due to gauge invariance, the supersymmetry of the operator appearing in equation (3.5) is restored on Nishimori's line. The integrand in (3.5) becomes symmetric, which is the very definition of a « zero dimensional integral » [8]. Nishimori's simplification therefore appears as a dimensional reduction.

\section{An interpretation of Nishimori's result for a general Ising spin glass.}

4.1 Some DEFINITIONS. - In all this section, we consider the Ising model on an arbitrary lattice with $N$ sites and $N_{\mathrm{B}}$ random bonds.

The quenched internal energy of the model can be separated, as in formula (3.1), into a regular contribution and a «potentially singular» one :

$$
\begin{aligned}
& U_{\mathrm{S}}= \\
& -\sum_{(i j)}\left|J_{i j}\left(1-t_{i j}^{2}\right) \frac{\sum_{\{S\}} S_{i} S_{j} \prod_{(k l) \neq(i j)}\left(1+t_{k l} S_{k} S_{l}\right)}{\sum_{\{S\}} \prod_{(k l)}\left(1+t_{k l} S_{k} S_{l}\right)}\right|
\end{aligned}
$$

where (ij) denotes an interacting pair of sites. Let us now consider a given bond $J_{01}=J_{1}$, and introduce the following function (the averages are taken over all bonds, except $J_{1}$ ) :

$$
\begin{aligned}
\mathcal{U}_{N}^{*}\left(J_{1}\right) & =-J_{1}\left(1-t_{1}^{2}\right) \times \\
& \times\left|\frac{\sum_{\{S\}} S_{0} S_{1} \prod_{(k l) \neq(01)}\left(1+t_{k l} S_{k} S_{l}\right)}{\sum_{\{S\}} \prod_{(k l)}\left(1+t_{k l} S_{k} S_{l}\right)}\right|^{*}
\end{aligned}
$$

All sites are supposed to be equivalent, and one has :

$$
U_{\mathrm{S}}=N_{\mathrm{B}} \int P\left(J_{1}\right) \mathcal{U}_{N}^{*}\left(J_{1}\right) \mathrm{d} J_{1} .
$$

This allows us to interpret $\mathcal{U}_{N}^{*}$ as a density of " singular » energy per frozen bond, the average being taken over all the other bonds.

For the one- and two-dimensional Ising model with nearest neighbour interactions considered previously, it is not difficult to see that the ratio $-\mathcal{U}_{N}^{*}(-J) / \mathcal{U}_{N}^{*}(J)$ is precisely the function $\rho_{N}(J)$ defined in section 3 . This is obvious in one dimension from equation (3.10): in two dimensions, where $\rho_{N}$ is defined by (3.8), it follows from the comparison between (4.2) and the supersymmetric formulation (2.20) of $U_{\mathrm{s}}$.

We thus define the following ratio of "singular" energies, for a general Ising spin glass :

$$
\rho_{N}(J)=-\frac{\mathcal{U}_{N}^{*}(-J)}{\mathcal{U}_{N}^{*}(J)} .
$$

4.2 WhAT HAPPENS ON NiSHIMORI's LINE. - We are now ready to give a more precise picture of Nishimori's result. In fact, what happens on Nishimori's line, defined by (3.1), is that the ratio $\rho_{N}(J)$ simplifies to become :

$$
\rho_{N}(J)=\mathrm{e}^{2 \beta J} .
$$

The proof is given in Appendix B, where the same result is derived for a similar function defined as :

$$
R_{N}(J)=-\frac{U_{N}^{*}(-J)}{U_{N}^{*}(J)}
$$

where $U_{N}^{*}(J)$ is the total singular energy when the bond $J$ is frozen. It is then clear from (4.3) that $U_{\mathrm{s}}$ vanishes, simply because (4.5) and (3.1) imply that the function $P(J) U_{N}^{*}(J)$ is odd under the change $J \rightarrow-J$, on the line. This shows that Nishimori's result can be understood as follows : the contribution to the «singular » energy of the system with one bond frozen to a given value $+J$ cancels on the average the same contribution with the bond frozen to the opposite value $-J$. The relation :

$$
\frac{P(J)}{P(-J)}=\rho_{N}(J)
$$

which holds on the line, clearly shows that Nishimori's result can be interpreted as a non-trivial relation between a local quantity involving the probability distribution over one bond, and a ratio of energies which depends on the whole system. Formula (4.5) illustrates the remarkable simplifications that can occur on the line for quantities which a priori have the full complexity of a spin glass problem.

It would have been extremely difficult to discover such a simplification by using perturbative methods, such as diagrammatic expansions for $U_{\mathrm{S}}, \rho_{N}$ or $R_{N}$. This is clearly apparent on the expansion (3.11) made in the one-dimensional case, where a global factorization of the series occurs. These properties can only become apparent through a global symmetry property, such as gauge invariance.

All these features are very similar to the properties of "disorder solutions" which occur for pure systems [10-12].

4. 3 FINITE SIZE SYSTEMS AND THE THERMODYNAMIC LIMIT. - As soon as condition (4.6) is met, (4.3) shows that $U_{\mathrm{S}}$ vanishes. Thus, it is a priori a more general condition than Nishimori's one, and it could have other solutions. 
The point is that Nishimori's condition satisfies (4.6) independently of the size of the system. Hence, it is interesting to study the behaviour of $\rho_{N}$ in the thermodynamic limit. This is most easily achieved in one dimension. For the sake of simplicity, let us consider the case of a binary distribution of bonds :

$P\left(J_{i, i+1}\right)=p \delta\left(J_{i, i+1}-J_{0}\right)+(1-p) \delta\left(J_{i, i+1}+J_{0}\right)$.

From (3.11), we obtain :

$$
\rho_{N}(J)=\frac{\left[\frac{2 p-1}{t_{0}}\right]^{N-1}+t}{\left[\frac{2 p-1}{t_{0}}\right]^{N-1}-t}
$$

so that its thermodynamic limit is :

$$
\left\{\begin{array}{l}
\rho_{\infty}(J)=\frac{1+t}{1-t}=\mathrm{e}^{2 \beta J} \text { on Nishimori's line } t_{0}=2 p-1 \\
\rho_{\infty}(J)=1 \quad \text { for } \quad\left|t_{0}\right|<2 p-1 \\
\rho_{\infty}(J)=-1 \text { for }\left|t_{0}\right|>2 p-1
\end{array}\right.
$$

(on the line $t_{0}=1-2 p, \rho_{N}$ has no limit). This result for $\rho_{\infty}$ is valid for a large class of laws $P$. This behaviour of $\rho_{N}$ should not surprise us, since neither $\rho_{N}$ nor $\mathcal{U}_{N}^{*}$ is an extensive quantity.

We thus see that if one imposes the condition (4.6) to be independent of $N$, Nishimori's condition is automatically satisfied. In this way the simplification of the quenched energy on the line seems to be associated with an invariance of the system respectively to its size.
This could have some links with the dimensional reduction of section 3 , and it would be interesting to study how these ideas apply in higher dimensions.

\section{Conclusion.}

In this paper, we have introduced a formulation which avoids the replica trick to express the quenched internal energy of the 2D Ising spin glass. This could provide a new method to study the critical properties of this model, using field theoretical techniques.

We have shown that Nishimori's result appears in this framework as a dimensional reduction due to a restoration of supersymmetry. The parameter which controls this restoration has been shown to have a simple physical interpretation for an Ising spin glass in any dimension. This allowed us to make the mechanism of Nishimori's simplification more precise.

In a companion paper, the role of gauge invariance will be further clarified by generalizing Nishimori's result to other groups than $Z_{2}$. There we shall also show how this result can be used to provide physical information on phase diagrams.

\section{Acknowledgments.}

We are grateful to Professor G. Toulouse and Professor N. Sourlas for their constant interest in our work. We are particularly indebted to J. M. Maillard for drawing our attention to Nishimori's result, for enlightening discussions and careful reading of the manuscript. We also thank J. P. Bouchaud for valuable comments.

\section{Appendix A.}

Here we explicitly construct the path integral representation of the quenched energy in dimension 2 . We start from an expression of $Z$ involving four independent fermions at each site $x_{k}, x_{k}^{+}, y_{k}, y_{k}^{+}[13,14]$

$$
Z=Z_{\mathrm{R}} \int \prod_{k} \mathrm{~d} x_{k} \mathrm{~d} x_{k}^{+} \mathrm{d} y_{k} \mathrm{~d} y_{k}^{+} \exp S
$$

where

$$
S=\sum_{k}\left(t_{k, k+1} x_{k}^{+} x_{k+1}+t_{k, k+2} y_{k}^{+} y_{k+2}+x_{k}^{+} y_{k}+y_{k}^{+} x_{k}+x_{k}^{+} y_{k}^{+}+x_{k} y_{k}+x_{k}^{+} x_{k}+y_{k}^{+} y_{k}\right)
$$

and $Z_{\mathrm{R}}$ is defined by formula (2.17).

In order to express $Z^{2}$, we introduce a new set of fermions at each site $u_{k}, u_{k}^{+}, v_{k}, v_{k}^{+}$, and define new fermionic variables :

$$
\begin{array}{ll}
a_{k}^{+}=\frac{1}{2}\left(x_{k}^{+}+x_{k}\right)+\frac{i}{2}\left(u_{k}^{+}+u_{k}\right) & c_{k}^{+}=\frac{1}{2}\left(u_{k}^{+}-u_{k}\right)+\frac{1}{2 i}\left(x_{k}^{+}-x_{k}\right) \\
b_{k}^{+}=\frac{1}{2}\left(y_{k}^{+}+y_{k}\right)+\frac{i}{2}\left(v_{k}^{+}+v_{k}\right) & d_{k}^{+}=\frac{1}{2}\left(v_{k}^{+}-v_{k}\right)+\frac{1}{2 i}\left(y_{k}^{+}-y_{k}\right) .
\end{array}
$$

We then obtain, after a straightforward calculation :

$$
Z^{2}=Z_{\mathrm{R}}^{2} \int D F \mathrm{e}^{S_{F}}
$$


with

$$
D F=\prod_{k} \mathrm{~d} a_{k} \mathrm{~d} a_{k}^{+} \mathrm{d} b_{k} \mathrm{~d} b_{k}^{+} \mathrm{d} c_{k} \mathrm{~d} c_{k}^{+} \mathrm{d} d_{k} \mathrm{~d} d_{k}^{+}
$$

and the fermionic action $S_{F}$ can be written in the compact form (2.18):

$$
S_{\mathrm{F}}=\sum_{k}\left(t_{k, k+1} K_{k, k+1}^{\mathrm{F}}+t_{k, k+2} K_{k, k+2}^{\mathrm{F}}+V_{k}^{\mathrm{F}}\right)
$$

with

$$
K_{k, k+1}^{\mathbf{F}}=\frac{1}{2}\left(a_{k}^{+} a_{k+1}-a_{k+1}^{+} a_{k}+c_{k}^{+} c_{k+1}-c_{k+1}^{+} c_{k}-i a_{k}^{+} c_{k+1}+i c_{k+1}^{+} a_{k}+i c_{k}^{+} a_{k+1}-i a_{k+1}^{+} c_{k}\right)
$$

$K_{k, k+2}^{\mathrm{F}}=$ same expression with $a_{k} \rightarrow b_{k}, c_{k} \rightarrow d_{k}, \mathbf{1} \rightarrow \mathbf{2}$

$V_{k}^{\mathrm{F}}=a_{k}^{+} b_{k}-b_{k}^{+} a_{k}-c_{k}^{+} d_{k}+d_{k}^{+} c_{k}+i\left(d_{k}^{+} a_{k}-a_{k}^{+} d_{k}+c_{k}^{+} b_{k}-b_{k}^{+} c_{k}+c_{k}^{+} a_{k}-\right.$

$$
\left.-a_{k}^{+} c_{k}+d_{k}^{+} b_{k}-b_{k}^{+} d_{k}\right) \text {. }
$$

As a result of these manipulations, $Z^{2}$ has the usual form of a Gaussian integral so that $Z^{-2}$ can be written as a free bosonic integral :

$$
Z^{-2}=Z_{\mathbf{R}}^{-2} \int D B \mathrm{e}^{S_{\mathbf{B}}}
$$

where B denotes collectively the bosonic variables $\alpha_{k}, \alpha_{k}^{*}, \beta_{k}, \beta_{k}^{*}, \gamma_{k}, \gamma_{k}^{*}, \delta_{k}, \delta_{k}^{*}$ and $S_{\mathrm{B}}$ is given by the same expression (A.4) as $S_{F}$, with the replacement

$$
\left\{\begin{array}{llll}
a_{k} \rightarrow \alpha_{k} & b_{k} \rightarrow \beta_{k} & c_{k} \rightarrow \gamma_{k} & d_{k} \rightarrow \delta_{k} \\
a_{k}^{+} \rightarrow \alpha_{k}^{*} & b_{k}^{+} \rightarrow \beta_{k}^{*} & c_{k}^{+} \rightarrow \gamma_{k}^{*} & d_{k}^{+} \rightarrow \delta_{k}^{*} .
\end{array}\right.
$$

This correspondence can be made rigourous provided the Hermitian part of the action $S_{\mathrm{B}}$ is shown to be negative definite.

As in the one-dimensional case, the action $S_{\mathrm{F}}+S_{\mathrm{B}}$ is supersymmetric under the same kinds of transformations as (2.13).

\section{Appendix B.}

In this appendix, we prove that on Nishimori's line, for a general Ising spin glass, one has :

$$
\rho_{N}(J)=\mathrm{e}^{2 \beta J} .
$$

According to (4.4), this reads :

$$
(1+t) u_{N}^{*}(J)+(1-t) u_{N}^{*}(-J)=0 .
$$

We use the fact that Nishimori's result applies to probability distributions which can be written as :

$$
P(J)=f(J) \mathrm{e}^{a J}
$$

where $f(J)$ is an even function of $J$, and $a$ is a coefficient. Nishimori's condition (1.1) then defines a subspace of the phase diagram of equation :

$$
a=\beta \text {. }
$$

We introduce a set $\left\{\sigma_{i}\right\}$ of Ising variables such that :

$$
\sigma_{0} \sigma_{1}=+1 \text {. }
$$

Using the form (B.3) for $P$ in equation (4.2), we perform the change of variables :

$$
J_{i j} \rightarrow \sigma_{i} \sigma_{j} J_{i j} .
$$

This leads to :

$$
u_{N}^{*}\left(J_{1}\right)=-J_{1}\left(1-t_{1}^{2}\right) \int_{-\infty}^{+\infty} \prod_{(i j)}^{*} f\left(J_{i j}\right) \mathrm{e}^{a J_{i j} \sigma_{l} \sigma_{j}} \mathrm{~d} J_{i j} \frac{\sum_{\{S\}} S_{0} S_{1} \prod_{(k l)}^{*}\left(1+t_{k l} \sigma_{k} S_{k} \sigma_{l} S_{l}\right)}{\sum_{\{S\}} \prod_{(k l)}\left(1+t_{k l} \sigma_{k} S_{k} \sigma_{l} S_{l}\right)} .
$$


We now make the reindexing $S_{k} \rightarrow \sigma_{k} S_{k}$ in all sums over $\{S\}$, and sum over all choices of $\{\sigma\}$ (having inserted the projector $\frac{1}{2}\left(1+\sigma_{0} \sigma_{1}\right)$ which takes condition (B.5) into account $)$. We obtain :

$$
\mathcal{U}_{N}^{*}\left(J_{1}\right)=-J_{1}\left(1-t_{1}^{2}\right) \frac{1}{2^{N}} \sum_{\{\sigma\}} \int \prod_{(i j)} * f\left(J_{i j}\right) \mathrm{e}^{a J_{i j} \sigma_{i} \sigma_{j}} \mathrm{~d} J_{i j}\left(1+\sigma_{0} \sigma_{1}\right) \frac{\sum_{\{S\}} S_{0} S_{1} \prod_{(k l)}^{*}\left(1+t_{k l} S_{k} S_{l}\right)}{\sum_{\{S\}} \prod_{(k l)}\left(1+t_{k l} S_{k} S_{l}\right)} .
$$

Making use of the following identities :

$$
\begin{gathered}
\mathrm{e}^{a J_{i j} \sigma_{i} \sigma_{j}}=\cosh a J_{i j}\left(1+\sigma_{i} \sigma_{j} \tanh a J_{i j}\right) \\
\left(1+t_{1}\right)\left(1+\sigma_{0} \sigma_{1}\right)=\left(1+\sigma_{0} \sigma_{1}\right)\left(1+t_{1} \sigma_{0} \sigma_{1}\right)
\end{gathered}
$$

formula (B.7) leads to :

$$
\begin{aligned}
\left(1+t_{1}\right) u_{N}^{*}\left(J_{1}\right)=-J_{1}(1- & \left.t_{1}^{2}\right) \frac{1}{2^{N}} \sum_{\{\sigma\}} \int_{(i j)} \prod^{*} f\left(J_{i j}\right) \cosh a J_{i j} \mathrm{~d} J_{i j}\left(1+\sigma_{0} \sigma_{1}\right) \times \\
& \times\left(1+t_{1} \sigma_{0} \sigma_{1}\right) \prod_{(k l)}^{*}\left(1+\sigma_{k} \sigma_{l} \tanh a J_{k l}\right) \frac{\sum_{\{S\}} S_{0} S_{1} \prod_{(k l)}^{*}\left(1+t_{k l} S_{k} S_{l}\right)}{\sum_{\{S\}} \prod_{(k l)}\left(1+t_{k l} S_{k} S_{l}\right)} .
\end{aligned}
$$

The corresponding expression of $\left(1-t_{1}\right) \mathcal{U}_{N}^{*}\left(-J_{1}\right)^{\prime}$ can be further transformed by making the following changes of variables in the integral and the sums over $\{S\}$ and $\{\sigma\}$ :

$$
S_{0} \rightarrow-S_{0}, \sigma_{0} \rightarrow-\sigma_{0}, t_{0 i} \rightarrow-t_{0 i}(i \neq 1) .
$$

The expression of $\left(1-t_{1}\right) U_{N}^{*}\left(-J_{1}\right)$ is thus obtained from (B.8) by simply replacing the term $\left(1+\sigma_{0} \sigma_{1}\right)$ by $\left(1-\sigma_{0} \sigma_{1}\right)$. Finally :

$$
\begin{aligned}
&\left(1+t_{1}\right) u_{N}^{*}\left(J_{1}\right)+\left(1-t_{1}\right) u_{N}^{*}\left(-J_{1}\right)=-J_{1}\left(1-t_{1}^{2}\right) \frac{1}{2^{N-1}} \int_{(i j)} \prod^{*} f\left(J_{i j}\right) \cosh a J_{i j} \mathrm{~d} J_{i j} \times \\
& \times \sum_{\{\sigma\}}\left(1+t_{1} \sigma_{0} \sigma_{1}\right) \prod_{(k l)}^{*}\left(1+\sigma_{k} \sigma_{l} \tanh a J_{k l}\right) \frac{\sum_{\{S\}} S_{0} S_{1} \prod_{(k l)}^{*}\left(1+t_{k l} S_{k} S_{l}\right)}{\sum_{\{S\}} \prod_{(k l)}\left(1+t_{k l} S_{k} S_{l}\right)} .
\end{aligned}
$$

On Nishimori's line, $a=\beta$ and a « miracle » occurs : the sum over $\{\sigma\}$ of the numerator becomes identical to the sum over $\{S\}$ of the denominator. Thus :

$$
\begin{aligned}
&\left(1+t_{1}\right) U_{N}^{*}\left(J_{1}\right)+\left(1-t_{1}\right) U_{N}^{*}(\left.-J_{1}\right)= \\
&=-J_{1}\left(1-t_{1}^{2}\right) \frac{1}{2^{N-1}} \int \prod_{(i j)}^{*} f\left(J_{i j}\right) \cosh \beta J_{i j} \mathrm{~d} J_{i j} \sum_{\{S\}} S_{0} S_{1} \prod_{(k l)}^{*}\left(1+t_{k l} S_{k} S_{l}\right) .
\end{aligned}
$$

This expression clearly vanishes, the integrand being odd under $J_{k l} \rightarrow-J_{k l}$. This establishes equation (B.2), and the desired result.

Let us mention that the equality (B.1) also applies, on Nishimori's line, to the function $R_{N}(J)$ defined by :

$$
R_{N}(J)=-\frac{U_{N}^{*}(-J)}{U_{N}^{*}(J)}
$$

$U_{N}^{*}(J)$ is the total singular energy when the bond $J$ is frozen, which reads (according to (4.1)) :

$$
U_{N}^{*}(J)=-\sum_{(i j)}\left|J_{i j}\left(1-t_{i j}^{2}\right) \frac{\sum_{\{\mathcal{S S \}}} S_{i} S_{j} \prod_{(k l) \neq(i j)}\left(1+t_{k l} S_{k} S_{l}\right)}{\sum_{\{S\}} \prod_{(k l)}\left(1+t_{k l} S_{k} S_{l}\right)}\right|^{*}
$$

The proof for $R_{N}$ is very similar to the one given above. 


\section{References}

[1] Edwards, S. F. and Anderson, P. W., J. Phys. F 5 (1975) 965.

[2] McKane, A. J., Phys. Lett. A 76 (1980) 22.

[3] Parisi, G. and Sourlas, N., J. Physique Lett. 41 (1980) L-403.

[4] Efetov, K. B., Adv. Phys. 32 (1983) 53.

[5] Nishimori, H., J. Phys. C 13 (1980) 4071.

[6] Nishimori, H., Prog. Theor. Phys. 66 (1981) 1169.

[7] Dotsenko, Vik. S. and Dotsenko, Vi. S., J. Phys. C 15 (1982) 485 ; Sov. Phys. JETP 56 (1982) 406.
[8] Parisi, G. and Sourlas, N., Phys. Rev. Lett. 43 (1979) 744.

[9] Parisi, G. and Sourlas, N., Nucl. Phys. B 206 (1982) 321.

[10] Stephenson, J., J. Math. Phys. 11 (1970) 420.

[11] Verhagen, A. M. W., J. Stat. Phys. 15 (1976) 219.

[12] Jaekel, M. T. and Maillard, J. M., J. Phys. A. 18 (1985) 1229. LPTENS $84 / 26$, to be published in J. Phys. A.

[13] Samuel, S., J. Math. Phys. 21 (1980) 2806.

[14] Jug, G., Phys. Rev. Lett. 53 (1984) 9. 